\title{
FACTORES DE RIESGO PARA HIPERTENSIÓN ARTERIAL EN POBLACIÓN ADULTA DE UNA REGIÓN URBANA DE ECUADOR
}

\author{
Rina Elizabeth Ortiz-Benavides ${ }^{1, a}$, Maritza Torres-Valdez ${ }^{2, b}$, Wilson Sigüencia-Cruz ${ }^{2, b}$, \\ Roberto Añez-Ramos ${ }^{3, c}$, Juan Salazar-Vílchez ${ }^{3, c}$, Joselyn Rojas-Quintero ${ }^{3, d}$, Valmore Bermúdez-Pirela ${ }^{3, e}$
}

RESUMEN

Objetivos. Determinar los factores de riesgo para hipertensión arterial (HTA) en población adulta residente en la ciudad de Cuenca, Ecuador. Materiales y métodos. Estudio transversal analítico realizado en adultos seleccionados mediante muestreo aleatorio multietápico, a quienes se les realizó evaluación clínica, evaluación antropométrica y de laboratorio. El diagnóstico de HTA fue definido según los criterios del JNC-7. Se realizó un modelo de regresión logística múltiple. Resultados. Se incluyeron 318 personas. La prevalencia de HTA fue de $25,8 \%$ (hombres: $27,2 \%$ vs mujeres: $24,7 \%$; $p=0,617)$. En el modelo de regresión logística múltiple, los factores de riesgo para HTA fueron la edad $>60$ años (OR 8,68; IC 95\%: 3,56-21,14; $p<0,001$ ), obesidad según índice de masa corporal (OR 2,36; IC 95\%: 1,04-5,70; $p=0,042)$, alto consumo calórico (OR 2,06; IC 95\%: 1,01-4,53; $p=0,044$ ) y el antecedente familiar de HTA (OR 1,58; IC 95\%: $1,02-2,90 ; p=0,040)$. Conclusiones. La presencia de HTA en esta población se asocia a factores intrínsecos como medioambientales, los cuales deben ser considerados en la evaluación rutinaria para la identificación y control temprano de esta enfermedad.

Palabras clave: Hipertensión; Obesidad; Factores de riesgo; Consumo de alimentos; Herencia. (fuente: DeCS BIREME).

\section{RISK FACTORS FOR ARTERIAL HYPERTENSION IN THE ADULT POPULATION OF AN URBAN REGION OF ECUADOR}

\begin{abstract}
Objectives. To determine the risk factors for arterial hypertension (HTN) in the adult resident population of the city of Cuenca, Ecuador. Materials and methods. A cross-sectional analytical study of adults selected via multistage random sampling who underwent clinical, anthropometric, and laboratory evaluations. The diagnosis of HTN was defined according to the JNC-7 criteria. A multiple logistic regression model was performed. Results. A total of 318 persons were included. The prevalence of HTN was $25.8 \%$ (males: $27.2 \%$ vs. females: $24.7 \%$; $p=0.617$ ). In the multiple logistic regression model, the risk factors for $\mathrm{HTN}$ were age $>60$ years (OR, 8.68; 95\% Cl, 3.56-21.14; $\mathrm{p}<0.001$ ), obesity according to body mass index (OR, 2.36; $95 \% \mathrm{Cl}, 1.04-5.70 ; \mathrm{p}=0.042)$, high caloric intake $(\mathrm{OR}, 2.06 ; 95 \% \mathrm{Cl}, 1.01-$ 4.53; $p=0.044)$, and family history of HTN $(\mathrm{OR}, 1.58 ; 95 \% \mathrm{Cl}, 1.02-2.90 ; p=0.040)$. Conclusions. The presence of HTN in this population is associated with both intrinsic and environmental factors, which should be considered in routine evaluations to ensure its early identification and control.
\end{abstract}

Keywords: Hypertension; Obesity; Risk factor; Food consumption; Heredity (source: MeSH NLM).

\section{INTRODUCCIÓN}

Entre los diversos factores de riesgo para las enfermedades cardiovasculares (ECV), la hipertensión arterial (HTA) es considerada uno de los más importantes a nivel mundial, asociándose a eventos coronarios y cerebrovasculares en aproximadamente 60 y $77 \%$ de los casos, respectivamente ${ }^{(1)}$. De igual forma, se estima que para el año 2030 la cifra de afectados por esta enfermedad ascienda en un $8 \%$. Una tendencia similar se ha observado en Ecuador durante la última década, con un ascenso en la incidencia de 256 a 1084 casos por cada 100 mil habitantes durante el periodo 20002009 (2); asimismo, un reporte previo ha determinado que específicamente en la ciudad de Cuenca, Ecuador la prevalencia de HTA se sitúa en un $25,8 \%{ }^{(3)}$.

\footnotetext{
Universidad Católica de Cuenca. Facultad de Psicología Clínica. Cuenca. Provincia del Azuay. República del Ecuador Ministerio de Salud Pública, Centro de Salud El Vale, Ciudad de Cuenca, Provincia del Azuay, República del Ecuador.

Centro de Investigaciones Endocrino - Metabólicas - “Dr. Félix Gómez”. Facultad de Medicina. La Universidad del Zulia. Maracaibo, Venezuela

Especialista en docencia universitaria, magíster en endocrinologia avanzada; ${ }^{b}$ Especialista en medicina familiar, magíster en endocrinologia avanzada ${ }^{c}$ Medico;

${ }^{\mathrm{d}}$ magíster en endocrinologia; ${ }^{\mathrm{e}}$ magíster en endocrinología, magíster en inmunologia, magíster en metabolismo humano, doctorado en ciencias medicas.

Recibido: 02/01/2016 Aprobado: 20/04/2016
}

Citar como: Ortiz-Benavides RE, Torres-Valdez M, Sigüencia-Cruz W, Añez-Ramos R, Salazar-Vílchez J, Rojas-Quintero J, Bermúdez-Pirela V. Factores de riesgo para hipertensión arterial en población adulta de una región urbana de Ecuador. Rev Peru Med Exp Salud Publica. 2016;33(2):248-55. doi: 10.17843/ rpmesp.2016.332.2214 
Considerando el origen de la HTA como primario (causa desconocida) y secundario a otros trastornos que condicionan la elevación de la presión arterial (PA) ${ }^{(4)}$, el análisis e identificación de potenciales factores de riesgo hallevado al estudio de numerosas variables, que pueden influir en la aparición y control de cifras de PA elevadas que tengan importantes implicaciones clínicas, entre las que figuran: edad, raza, tabaquismo, antecedente familiar, menopausia, hábitos nutricionales, obesidad, entre muchos otros ${ }^{(5,6)}$. El manejo integral de la HTA debe abarcar tanto medidas farmacológicas como no farmacológicas, además, en numerosas oportunidades nos encontramos con pacientes hipertensos que presentan un conjunto de comorbilidades que deben, de igual manera, tratarse simultáneamente ${ }^{(7)}$; por ende, la correcta evaluación de este numeroso grupo de sujetos debe incluir la identificación de esos factores ambientales acompañantes.

Ante la importancia epidemiológica que representa la HTA, así como la necesidad de identificar sus principales determinantes, el objetivo del estudio fue determinar los factores de riesgo para HTA en una población de adultos de la ciudad de Cuenca, Ecuador.

\section{MATERIALES Y MÉTODOS}

\section{DISEÑO Y POBLACIÓN DE ESTUDIO}

Se realizó un estudio transversal analítico en la región urbana de Cuenca, la cual está dividida en 16 parroquias: San Sebastián, Bellavista, El Batán, Yanuncay, Sucre, Huayna Capac, Gil Ramírez Dávalos, Sagrario, San Blas, El Vecino, Cañaribamba, Totoracocha, Monay, Machangara, y Hermano Miguel, con una población total de 329928 habitantes (se sumó a estos un grupo de 1960 individuos que habitan parroquias rurales en el límite con las parroquias urbanas) para un total de 331888 habitantes, los población mayor de 18 años que habita el área urbana de la ciudad de cuenca fue de 223798 individuos, para estas estimaciones fueron utilizadas las valoraciones demográficas obtenidas del censo 2010 realizado por el Instituto Nacional de Estadísticas y Censos (INEC).

El cálculo del tamaño de la muestra para cada parroquia se realizó de forma proporcional, mediante un muestreo aleatorio multietápico por conglomerados, donde cada conglomerado estuvo representado por cada una de las 16 parroquias, obteniéndose un tamaño muestral de 318 individuos mayores de 18 años. El criterio de inclusión fue todos los habitantes mayores de 18 años. Dentro de los criterios de exclusión se consideraron a las mujeres en periodo de gestación, individuos recluidos en instituciones penales, hospitales, o cuarteles militares.

Se llevó a cabo un muestreo aleatorio multietápico por conglomerados durante el periodo octubre 2013 a febrero 2014, donde cada uno de los conglomerados estuvo representado por manzanas y a su vez por viviendas las cuales se escogieron al azar manejando la información del censo de la ciudad de cuenca. La tasa de respuesta de los participantes fue de un $98 \%$, donde los sujetos no participantes indicaron no desear participar en el estudio por negarse a ser sometidos a exámenes clínicos o antropométricos; por lo tanto, se realizó un sobremuestreo hasta completar los casos contemplados en la muestra determinada.

\section{EVALUACIÓN DE LOS INDIVIDUOS}

A todos los individuos que participaron en el estudio se les realizó historia clínica completa evaluándose la presencia de antecedentes personales de HTA o diabetes mellitus tipo 2 (DM2). El grupo étnico se evaluó fenotípicamente clasificando a los individuos como mestizo, blanco y otros (negro, mulato, montubio). Se evaluó el estatus socioeconómico mediante la escala de Graffar modificado por Méndez-Castellano ${ }^{(8)}$ que estratifica a los sujetos en cinco estratos: clase alta (estrato I); clase media alta, (estrato II); clase media (estrato III), clase obrera (estrato IV), y extrema pobreza (estrato V).

\section{HÁBITOS PSICOBIOLÓGICOS}

Se interrogó el consumo de alcohol como autorreporte según la historia clínica en consumidor y no consumidor ${ }^{(9)}$. Por otra parte, fue evaluado el hábito tabáquico, categorizándose a los individuos en no fumadores, fumadores, y exfumadores a aquellos con un año o más sin consumir ningún tipo de tabaco ${ }^{(10)}$

\section{EVALUACIÓN DE ACTIVIDAD FÍSICA}

Se realizó el cuestionario internacional de actividad física, el cual fue diseñado para la medición de la actividad física en cuatro dominios: trabajo, transporte, actividades del hogar (jardinería y otros) y ocio (tiempo libre, recreación o ejercicio) ${ }^{(11)}$. El formato largo del IPAQ (IPAQ-LF) contiene preguntas correspondientes a la frecuencia y duración de la caminata (actividad leve), actividades moderadas o actividad vigorosas de por lo menos $10 \mathrm{~min}$ de duración. Los minutos/ semanas de actividad leve, moderada o vigorosa son convertidos a sus equivalentes metabólicos (MET), para así determinar el consumo energético. Los datos se calcularon de acuerdo al resultado MET promedio en cada actividad y a partir de estas consideraciones se realizó el Scoring IPAQ para determinar los patrones de actividad física que son reportados como: actividad física alta, moderada o baja ${ }^{(11)}$; dentro de los análisis del IPAQ fueron excluidos ocho individuos, ya que no cumplieron con los criterios de depuración del IPAQ durante los análisis de determinación del patrón de actividad física a través del Scoring IPAQ. Además, se analizó la actividad 
física expresada en $\mathrm{MET} / \mathrm{min} / \mathrm{sem}$ para el dominio de actividad física de ocio del IPAQ, el cual ha demostrado tener un papel como factor protector cardiovascular (12), para los análisis del estudio "actividad física de ocio" fue reclasificada en terciles obteniéndose la siguiente clasificación: mujeres (ninguna: $0 \mathrm{MET} / \mathrm{min} / \mathrm{sem}$; leve: <33 MET/min/sem; moderada: 33-346,49; alta: $\geq 346,5$ $\mathrm{MET} / \mathrm{min} / \mathrm{sem}$ ); hombres (ninguna: $0 \mathrm{MET} / \mathrm{min} / \mathrm{sem}$; leve: <66; MET/min/sem; moderada: 66-1046,61; Alta: $\geq 1046,62 \mathrm{MET} / \mathrm{min} / \mathrm{sem}$ ).

\section{EVALUACIÓN ANTROPOMÉTRICA}

Se determinó el peso y la talla de los individuos mediante el uso de balanza-tallímetro Health o Meter Professional (USA) con capacidad $180 \mathrm{~kg}$, para lo cual cada sujeto fue evaluado de pie, en posición erguida, sin calzado ni vestimenta. Los individuos se clasificaron ponderalmente mediante los puntos de corte del índice de masa corporal (IMC) sugeridos por la OMS ${ }^{(13)}$. La circunferencia abdominal se midió con una cinta métrica metálica Rosscraft, USA, calibrada en milímetros y centímetros, a la altura de la línea media axilar en el punto imaginario que se encuentra entre la parte inferior de la última costilla y el punto más alto de la cresta iliaca, de posición de pie, al final de una espiración (14).

\section{EVALUACIÓN NUTRICIONAL}

Para la evaluación nutricional se aplicó el recordatorio de $24 \mathrm{~h}$, el cual recogió datos de la ingesta de alimentos el día anterior tanto en el desayuno, almuerzo, cena y sus respectivas meriendas, los cuales fueron analizados por un equipo nutricionista para la determinación del consumo diario de calorías (kcal/día). Las calorías consumidas fueron reclasificadas en terciles: tercil 1: $<1960,94 \mathrm{kcal} / \mathrm{día}$; tercil 2: 1960,94-2471,93 kcal/día; tercil 3: $\geq 2471,94 \mathrm{kcal} / \mathrm{día}$.

\section{EVALUACIÓN DE LA PRESIÓN ARTERIAL}

La determinación de la presión arterial fue realizada por un equipo de ocho médicos especialistas en medicina familiar y con capacitación para realizar la adecuada técnica de medición por el método auscultatorio, para lo que se utilizó un esfigmomanómetro calibrado y validado. Se le midió al paciente sentado y quieto por lo menos $15 \mathrm{~min}$ con los pies en el suelo y el brazo a la altura del corazón, siendo la presión arterial sistólica el punto en el que se escuchó el primero de dos o más sonidos (fase 1) y la presión arterial diastólica es el punto en el que desapareció el sonido (fase 5). Se verificó la PA por tres ocasiones (luego de $10 \mathrm{~min}$ de descanso) y se realizó un promedio de las tomas. El diagnóstico de HTA se realizó de dos formas: a) autorreporte como antecedente personal; y b) diagnóstico durante la evaluación clínica, calificándose acorde con los criterios del Comité Norteamericano para la Prevención, Detección, Evaluación y Tratamiento de la HTA en su VII informe (JNC-7) en dos ocasiones separadas ${ }^{(15)}$.

\section{ANÁLISIS DE LABORATORIO}

Se recogieron muestras de sangre (en tubos Vacutainer sin anticoagulante) después de $10 \mathrm{~h}$ de ayuno, el suero se obtuvo por centrifugación. Se determinaron los niveles de triglicéridos (TAG), colesterol HDL, colesterol LDL y glucosa plasmática mediante el equipo Mindray b 88, semiaulant. Para la determinación de glicemia, colesterol total, triglicéridos, colesterol LDL se utilizó un kit enzimático-colorimétrico de (Reaactlab); para la cuantificación de colesterol HDL se utilizó un kit enzimático-colorimétrico comercial (Human Frisonex).

\section{VARIABLES METABÓLICAS Y OBESIDAD}

Las variables metabólicas y la obesidad abdominal se definieron de acuerdo a los criterios sugeridos por el consenso realizado por la IDF/AHA/NHLBI/WHF/IAS/ IASO (2009) ${ }^{(16)}$. Según la circunferencia abdominal la obesidad fue clasificada en $\geq 90 \mathrm{~cm}$ para hombres y $\geq 80$ $\mathrm{cm}$ para mujeres. TAG elevado: $\geq 150 \mathrm{mg} / \mathrm{dL}$; colesterol HDL bajo: hombres $<40 \mathrm{mg} / \mathrm{dL}$, mujeres <50 mg/dL; glicemia elevada: $\geq 100 \mathrm{mg} / \mathrm{dL}$, o el uso de medicamentos para el control de alguna alteración respectiva.

\section{ANÁLISIS ESTADÍSTICO}

Las variables cualitativas fueron presentadas como frecuencias absolutas y relativas; la prueba $Z$ se utilizó para comparar las proporciones entre grupos y la prueba de chi cuadrado para determinar la asociación entre variables cualitativas. Para evaluar la distribución normal de las variables cuantitativas se utilizó la prueba de Kolmogorov Smirnov. Las variables con distribución normal fueron expresadas con la media \pm desviación estándar. Se realizó un modelo de regresión logística bivariado para la estimación de odds ratio (IC 95\%) para HTA y multivariable ajustado por sexo, grupo etario, grupo étnico, estrato socioeconómico, hábito tabáquico y alcohólico, antecedente familiar de HTA, categorías de IMC, circunferencia abdominal alta, HDL-C baja, TAG alto, glicemia elevada, terciles del consumo diario de calorías y patrones de actividad física. Los datos obtenidos fueron analizados mediante el paquete informático SPSS versión 20, para Windows (SPSS Inc. Chicago, IL), se consideraron resultados significativos cuando el valor $p$ fue menor de 0,05.

\section{CONSIDERACIONES ÉTICAS}

El protocolo del estudio fue evaluado y aprobado por el comité de ética del Centro de Investigaciones Endocrino- 
Metabólicas "Dr. Félix Gómez" de la Universidad del Zulia, como parte del desarrollo conjunto de una red de investigación multidisciplinaria para evaluar alteraciones endocrino-metabólicas en la ciudad de Cuenca, Ecuador. Los individuos que participaron en el estudio firmaron un consentimiento informado en el cual se expuso el respeto a la vida, la salud, la confidencialidad, la intimidad, la autonomía y la dignidad; asimismo, se les explicó todos los detalles concernientes al estudio y los procedimientos a los cuales iban a ser sometidos, antes de realizarles el examen clínico, físico y de laboratorio.

\section{RESULTADOS}

La muestra estuvo conformada por 318 individuos de los cuales el $57,2 \%(n=182)$ correspondió al sexo femenino. La edad media de la población general fue de 42,8 \pm 15,5 años. Los grupos etarios más prevalentes fueron el de menos de 40 años con $44,7 \%$, seguido del grupo de 40 a 59 años $(39,0 \%)$ y 60 años o más con $16,4 \%$. El grupo de individuos mestizos fue el grupo étnico más prevalente con un 96,2\% $(n=306)$. El resto de características generales se muestra en la Tabla 1.

La prevalencia total de HTA fue de $25,8 \%(n=82)$, el $14,8 \%(n=47)$ de la población presentó HTA conocida y un $11,0 \%(n=35)$ de la población fue diagnosticado como hipertenso durante el estudio. Al evaluar según sexo, se observó una prevalencia de HTA de $24,7 \% \quad(n=45)$ en las mujeres y un $27,2 \%(n=37)$ en los hombres. La prevalencia de HTA según los grupos etarios, variables antropométricas y sociodemográficas se muestra en la Tabla 2, donde se muestra una asociación significativa entre el grupo etario y la $\operatorname{HTA}(p<0,001)$, con un aumento en la prevalencia de HTA a medida que se incrementa la edad, desde un $12,0 \%$ en el grupo de menos de 40 años hasta un $53,8 \%$ en el grupo de 60 años y más. Por otra parte, se observó una asociación entre la HTA y el estatus socioeconómico $(p=0,001)$, con un aumento de la frecuencia de hipertensos a medida se incrementa el estrato (a partir del estrato II: $18,6 \%$; estrato III: $19,8 \%$; estrato IV: $27,5 \%$ y estrato V: $70,0 \%$ ); no se evidenció una asociación significativa con el grupo étnico, condición laborar, patrones de actividad física o actividad física realizada en el tiempo de ocio por los sujetos (Tabla 2).

Por otra parte, la presencia de antecedente familiar de HTA mostró una asociación significativa con la HTA, con un $30,9 \%$ de prevalencia de HTA aquellos sujetos con familiares de primer grado hipertensos $(p<0,05)$. La clasificación según IMC también mostró asociación con la presencia de HTA $(p<0,001)$ con una tendencia al aumento del porcentaje de HTA a medida que se incrementó de categoría de IMC, el grupo de menos de $25 \mathrm{~kg} / \mathrm{m}^{2}$ : 16,4\% ( $\left.\mathrm{n}=19\right) ; 25-29 \mathrm{~kg} / \mathrm{m}^{2}(22,8 \% ; \mathrm{n}=29)$ y $\geq 30 \mathrm{~kg} / \mathrm{m}^{2}$ (45,3\%; $\mathrm{n=75)}$. Asimismo la HTA mostró una asociación con la presencia de obesidad abdominal $(p=0,010)$ (Tabla 2).

En el modelo de regresión multivariable para la presencia de HTA, los factores asociados fueron el grupo de edad de 40-59 años (OR 2,66; IC 95\%: 1,25-5,65; $p=0,010$ ) y $\geq 60$ años (OR: 8,68; IC 95\%: 1,25-21,14; $p<0,001)$. Seguido de la presencia de obesidad por IMC (OR 2,36; IC 95\%: $1,04-5,70 ; p=0,042)$. El resto de factores asociados fueron el alto consumo calórico y el antecedente familiar de HTA. Al analizar el consumo calórico como predictor de HTA, se observó que los sujetos con un consumo mayor o igual a $2471,94 \mathrm{kcal} / 24 \mathrm{~h}$ mostraron 2,06 veces mayor riesgo para HTA que los sujetos con un consumo menor a 1960,94 kcal/24 h (OR 2,06; IC 95\%: 1,01-4,53; $p=0,044)$ y los individuos con antecedente familiar de HTA presentaron $58 \%$ más riesgo para HTA que los sujetos sin antecedente de hipertensión (OR 1,58; IC 95\%: 1,02-2,90; $p=0,040$ ) (Tabla 2).

\section{DISCUSIÓN}

En el presente estudio se puede destacar que para la población de Cuenca los factores que ejercen mayor influencia para hipertensión arterial son la edad por encima de los 40 años, la presencia de obesidad de acuerdo al IMC, el antecedente familiar de HTA y la elevada ingesta calórica; por lo tanto, estos factores deben de ser abordados en la población para disminuir el riesgo a desarrollar HTA. Una limitante del presente estudio fue que no determinó la ingesta de sal como posible factor de riesgo para hipertensión arterial.

Los factores relacionados a la elevación de las cifras de PA varían entre las diferentes poblaciones y abarcan desde variables sociodemográficas hasta alteraciones específicas como trastornos endocrinos, renales e, incluso, consumo de ciertas sustancias o fármacos ${ }^{(17)}$, la importancia en la identificación de estos factores radica en el potencial enfoque terapéutico cuando el trastorno o la sustancia es la causa desencadenante de la HTA, o cuando estas características acompañan a la elevación de las cifras de PA, interviniendo en su mecanismo fisiopatológico o incluso influyendo en la adherencia al tratamiento ${ }^{(18)}$.

Es ampliamente conocido que el envejecimiento está acompañado de una serie de cambios metabólicos y degenerativos que afectan diversos órganos incluyendo la pared vascular (19), cuya rigidez y aumento progresivo de la resistencia a nivel periférico constituyen determinantes de suma importancia en el desarrollo o progresión de la HTA en los grupos etarios más longevos. Este comportamiento biológico hace necesario la exploración sistemática de este trastorno 
Tabla 1. Características generales de la población adulta de Cuenca, Ecuador

\begin{tabular}{|c|c|c|c|c|c|c|}
\hline & \multicolumn{2}{|c|}{ Femenino } & \multicolumn{2}{|c|}{ Masculino } & \multicolumn{2}{|c|}{ Total } \\
\hline & $n=182$ & $(\%)$ & $n=136$ & $(\%)$ & $\mathrm{N}=318$ & $(\%)$ \\
\hline \multicolumn{7}{|l|}{ Grupos etarios } \\
\hline$<40$ años & 84 & $(46,2)$ & 58 & $(42,6)$ & 142 & $(44,7)$ \\
\hline $40-59$ años & 68 & $(37,4)$ & 56 & $(41,2)$ & 124 & $(39,0)$ \\
\hline 60 años o más & 30 & $(16,5)$ & 22 & $(16,2)$ & 52 & $(16,4)$ \\
\hline \multicolumn{7}{|l|}{ Condición laboral } \\
\hline Empleado & 76 & $(41,8)$ & 76 & $(55,9)$ & 152 & $(47,8)$ \\
\hline Desempleado & 46 & $(25,3)$ & 20 & $(14,7)$ & 66 & $(20,8)$ \\
\hline Subempleado & 60 & $(33,0)$ & 40 & $(29,4)$ & 100 & $(31,4)$ \\
\hline \multicolumn{7}{|l|}{ Grupos étnicos } \\
\hline Mestizo & 178 & $(97,8)$ & 128 & $(94,1)$ & 306 & $(96,2)$ \\
\hline Blanco & 3 & $(1,6)$ & 5 & $(3,7)$ & 8 & $(2,5)$ \\
\hline Otros & 1 & $(0,5)$ & 3 & $(2,2)$ & 4 & $(1,3)$ \\
\hline \multicolumn{7}{|c|}{ Estrato socioeconómico } \\
\hline Estrato I & 0 & - & 2 & $(1,5)$ & 2 & $(0,6)$ \\
\hline Estrato II & 15 & $(8,2)$ & 28 & $(20,6)$ & 43 & $(13,5)$ \\
\hline Estrato III & 60 & $(33,0)$ & 36 & $(26,5)$ & 96 & $(30,2)$ \\
\hline Estrato IV & 103 & $(56,6)$ & 64 & $(47,1)$ & 167 & $(52,5)$ \\
\hline Estrato V & 4 & $(2,2)$ & 6 & $(4,4)$ & 10 & $(3,1)$ \\
\hline \multicolumn{7}{|l|}{ Hábito tabáquico } \\
\hline No & 161 & $(88,5)$ & 53 & $(39,0)$ & 214 & $(67,3)$ \\
\hline Sí & 11 & $(6,0)$ & 49 & $(36,0)$ & 60 & $(18,9)$ \\
\hline Fumó en el pasado & 10 & $(5,5)$ & 34 & $(25,0)$ & 44 & $(13,8)$ \\
\hline \multicolumn{7}{|l|}{ Consumo de alcohol } \\
\hline No & 156 & $(85,7)$ & 83 & $(61,0)$ & 239 & $(75,2)$ \\
\hline Sí & 26 & $(14,3)$ & 53 & $(39,0)$ & 79 & $(24,8)$ \\
\hline \multicolumn{7}{|c|}{ Antecedente familiar de HTA } \\
\hline No & 88 & $(48,4)$ & 78 & $(57,4)$ & 166 & $(52,2)$ \\
\hline Sí & 94 & $(51,6)$ & 58 & $(42,6)$ & 152 & $(47,8)$ \\
\hline \multicolumn{7}{|c|}{ Índice de masa corporal } \\
\hline$<25 \mathrm{~kg} / \mathrm{m}^{2}$ & 66 & $(36,3)$ & 50 & $(36,8)$ & 116 & $(36,5)$ \\
\hline $25-29 \mathrm{~kg} / \mathrm{m}^{2}$ & 66 & $(36,3)$ & 61 & $(44,9)$ & 127 & $(39,9)$ \\
\hline$\geq 30 \mathrm{~kg} / \mathrm{m}^{2}$ & 50 & $(27,5)$ & 25 & $(18,4)$ & 75 & $(23,6)$ \\
\hline \multicolumn{7}{|l|}{ Colesterol HDL bajo* } \\
\hline No & 40 & $(22,0)$ & 58 & $(42,6)$ & 98 & $(30,8)$ \\
\hline Sí & 142 & $(78,0)$ & 78 & $(57,4)$ & 220 & $(69,2)$ \\
\hline \multicolumn{7}{|c|}{ Triacilglicéridos altos* } \\
\hline No & 112 & $(61,5)$ & 74 & $(54,4)$ & 186 & $(58,5)$ \\
\hline Sí & 70 & $(38,5)$ & 62 & $(45,6)$ & 132 & $(41,5)$ \\
\hline \multicolumn{7}{|c|}{ Obesidad abdominal* $^{*}$} \\
\hline No & 28 & $(15,4)$ & 34 & $(25,0)$ & 62 & $(19,5)$ \\
\hline Sí & 154 & $(84,6)$ & 102 & $(75,0$ & 256 & $(80,5)$ \\
\hline \multicolumn{7}{|l|}{ Glicemia elevada* } \\
\hline No & 149 & $(81,9)$ & 111 & $(81,6)$ & 260 & $(81,8)$ \\
\hline Sí & 33 & $(18,1)$ & 25 & $(18,4$ & 58 & $(18,2)$ \\
\hline \multicolumn{7}{|c|}{ Patrones de actividad física (IPAQ) } \\
\hline Baja & 13 & $(7,5)$ & 18 & $(14,3)$ & 31 & $(10,4)$ \\
\hline Moderada & 55 & $(31,8)$ & 37 & $(29,4)$ & 92 & $(30,8)$ \\
\hline Alta & 105 & $(60,7)$ & 71 & $(56,3)$ & 176 & $(58,9)$ \\
\hline Actividad física en 0 & & & & & & \\
\hline Ninguna & 72 & $(41,6)$ & 34 & $(27,0)$ & 106 & $(35,5)$ \\
\hline Leve & 32 & $(18,5)$ & 29 & $(23,0)$ & 61 & $(20,4)$ \\
\hline Moderada & 35 & $(20,2)$ & 32 & $(25,4)$ & 67 & $(22,4)$ \\
\hline Alta & 34 & $(19,7)$ & 31 & $(24,6)$ & 65 & $(21,7)$ \\
\hline Terciles de calorías & & & & & & \\
\hline Tercil 1 & 77 & $(42,3)$ & 28 & $(20,6)$ & 105 & $(33,0)$ \\
\hline Tercil 2 & 56 & $(30,8)$ & 49 & $(36,0)$ & 105 & $(33,0)$ \\
\hline Tercil 3 & 49 & $(26,9)$ & 59 & $(43,4)$ & 108 & $(34,0)$ \\
\hline
\end{tabular}

" Consenso de IDF/NHLBI/AHA-2009. HTA: hipertensión arterial. Actividad física en ocio (IPAQ): mujeres (ninguna: 0 MET/min/sem; Leve: <33 MET/min/sem; moderada: 33346,49; alta: $\geq 346,5 \mathrm{MET} / \mathrm{min} / \mathrm{sem}$ ); hombres (ninguna: $0 \mathrm{MET} / \mathrm{min} / \mathrm{sem}$; leve: <66; MET/min/sem; moderada: 66-1046,61; alta: $\geq 1046,62 \mathrm{MET} / \mathrm{min} / \mathrm{sem}$ ). 
Tabla 2. Modelo de regresión logística de los factores de riesgo para hipertensión arterial en la población adulta de Cuenca, Ecuador

\begin{tabular}{|c|c|c|c|c|c|c|c|c|}
\hline & \multicolumn{4}{|c|}{ Hipertensión arterial } & \multirow[b]{2}{*}{$\begin{array}{c}\text { Análisis } \\
\text { bivariado }\end{array}$} & \multirow[b]{3}{*}{ Valor $p$} & \multirow{3}{*}{$\begin{array}{c}\begin{array}{c}\text { Análisis } \\
\text { multivariable }\end{array} \\
\text { OR (IC 95\%) }\end{array}$} & \multirow[b]{3}{*}{ Valor $p$} \\
\hline & \multicolumn{2}{|c|}{ Ausente } & \multicolumn{2}{|c|}{ Presente } & & & & \\
\hline & $\mathbf{n}$ & $\%$ & $\mathbf{n}$ & $\%$ & OR (IC 95\%) & & & \\
\hline \multicolumn{9}{|l|}{ Sexo } \\
\hline Femenino & 137 & 75,3 & 45 & 24,7 & 1,00 & - & 1,00 & - \\
\hline Masculino & 99 & 72,8 & 37 & 27,2 & $1,13(0,68-1,88)$ & 0,612 & $0,94(0,45-1,97)$ & 0,888 \\
\hline \multicolumn{9}{|l|}{ Grupos etarios } \\
\hline$<40$ años & 125 & 88,0 & 17 & 12,0 & 1,00 & - & 1,00 & - \\
\hline 40-59 años & 87 & 70,2 & 37 & 29,8 & $3,12(1,65-5,90)$ & $<0,001$ & $2,66(1,25-5,65)$ & 0,001 \\
\hline$\geq 60$ años & 24 & 46,2 & 28 & 53,8 & $4,07(4,07-18,05)$ & $<0,001$ & $8,68(3,56-21,14)$ & $<0,001$ \\
\hline \multicolumn{9}{|l|}{ Hábito tabáquico } \\
\hline No & 163 & 76,2 & 51 & 23,8 & 1,00 & - & 1,00 & - \\
\hline Fumador & 45 & 75,0 & 15 & 25,0 & $1,06(0,54-2,06)$ & 0,855 & $0,82(0,34-1,99)$ & 0,677 \\
\hline Exfumador & 28 & 63,6 & 16 & 36,4 & $1,82(0,91-3,64)$ & 0,082 & $1,49(0,626-3,58)$ & 0,364 \\
\hline \multicolumn{9}{|l|}{ Hábito alcohólico } \\
\hline No & 179 & 74,9 & 60 & 25,1 & 1,00 & - & 1,00 & - \\
\hline Sí & 57 & 72,2 & 22 & 27,8 & $1,15(0,65-2,04)$ & 0,62 & $1,35(0,64-2,85)$ & 0,422 \\
\hline \multicolumn{9}{|l|}{ Antecedente familiar de HTA } \\
\hline No & 131 & 78,9 & 35 & 21,1 & 1,00 & - & 1,00 & - \\
\hline Sí & 105 & 69,1 & 47 & 30,9 & $1,67(1,00-2,78)$ & 0,042 & $1,58(1,02-2,90)$ & 0,040 \\
\hline \multicolumn{9}{|l|}{ Índice de masa corporal } \\
\hline$<25 \mathrm{~kg} / \mathrm{m}^{2}$ & 97 & 83,6 & 19 & 16,4 & 1,00 & - & 1,00 & - \\
\hline $25-29 \mathrm{~kg} / \mathrm{m}^{2}$ & 98 & 77,2 & 29 & 22,8 & $2,78(1,57-4,92)$ & $<0,001$ & $1,19(0,78-2,09)$ & 0,834 \\
\hline$\geq 30 \mathrm{~kg} / \mathrm{m}^{2}$ & 41 & 54,7 & 34 & 45,3 & $4,18(2,27-7,69)$ & $<0,001$ & $2,36(1,04-5,70)$ & 0,042 \\
\hline \multicolumn{9}{|l|}{ Colesterol HDL bajo* } \\
\hline No & 71 & 72,4 & 27 & 27,6 & 1,00 & - & 1,00 & - \\
\hline Sí & 165 & 75,0 & 55 & 25,0 & $0,87(0,51-1,50)$ & 0,630 & $0,78(0,39-1,56)$ & 0,493 \\
\hline \multicolumn{9}{|l|}{ Triglicéridos altos* } \\
\hline No & 142 & 76,3 & 44 & 23,7 & 1,00 & - & 1,00 & - \\
\hline Sí & 94 & 71,2 & 38 & 28,8 & $1,30(0,78-2,16)$ & 0,302 & $1,08(0,57-2,05)$ & 0,810 \\
\hline \multicolumn{9}{|l|}{ Obesidad abdominal* } \\
\hline No & 54 & 87,1 & 8 & 12,9 & 1,00 & - & 1,00 & - \\
\hline Sí & 182 & 71,1 & 74 & 28,9 & $2,74(1,24-6,04)$ & 0,001 & $1,27(0,42-3,82)$ & 0,663 \\
\hline \multicolumn{9}{|l|}{ Glicemia elevada* } \\
\hline No & 196 & 75,4 & 64 & 24,6 & 1,00 & - & 1,00 & - \\
\hline Sí & 40 & 69,0 & 18 & 31,0 & $1,37(0,73-2,57)$ & 0,310 & $0,81(0,38-1,73)$ & 0,592 \\
\hline \multicolumn{9}{|c|}{ Patrones de actividad física (IPAQ) } \\
\hline Baja & 23 & 74,2 & 8 & 25,8 & 1,00 & - & 1,00 & - \\
\hline Moderada & 67 & 72,8 & 25 & 27,2 & $1,07(0,42-2,70)$ & 0,880 & $0,98(0,38-3,26)$ & 0,827 \\
\hline Alta & 134 & 76,1 & 42 & 23,9 & $0,90(0,37-2,16)$ & 0,812 & $0,84(0,30-2,30)$ & 0,735 \\
\hline \multicolumn{9}{|c|}{ Calorías consumidas (kcal/24 h) } \\
\hline Tercil $1(<1960,94)$ & 85 & 81,0 & 20 & 19,0 & 1,00 & - & 1,00 & - \\
\hline Tercil $2(1960,94-2471,93)$ & 77 & 73,3 & 28 & 26,7 & $1,54(0,80-2,96)$ & 0,192 & $1,77(0,81-3,89)$ & 0,143 \\
\hline Tercil $3(\geq 2471,94)$ & 74 & 68,5 & 34 & 31,5 & $1,95(1,03-3,68)$ & 0,030 & $2,06(1,01-4,53)$ & 0,044 \\
\hline
\end{tabular}

HTA: hipertensión arterial; * Consenso de IDF/NHLBI/AHA-2009

en la población adulta mayor, al observarse tendencias similares en diversas regiones del mundo ${ }^{(20-23)}$.

Asimismo, estos hallazgos demuestran la presencia de factores intrínsecos y medioambientales asociados a un mayor riesgo de presentar HTA; previamente, se ha planteado que el antecedente familiar de esta alteración es un predictor importante en la aparición de cifras elevadas de PA ${ }^{24)}$, relacionado a diversos trastornos moleculares y fisiológicos que abarcan desde cambios en la elasticidad arterial (25) hasta una activación simpática desde edades tempranas ${ }^{(26)}$.

Entre los diversos factores ambientales evaluados, se observa que aquellos relacionados con el balance energético fueron los de mayor relevancia, siendo 
la obesidad determinada por IMC y el alto consumo calórico los más asociados a la presencia de HTA. En cuanto a la obesidad, nuestros resultados se asemejan a los obtenidos por Amira et al. (27), en sujetos de una comunidad urbana de Nigeria donde observaron que los pacientes obesos mostraban 2,6 veces más riesgo de ser hipertensos en comparación con los normopeso; sin embargo, a diferencia de nuestros hallazgos los individuos con sobrepeso también mostraron un mayor riesgo. Esto demuestra la importancia de intervenir de forma temprana en la población con sobrepeso de Cuenca, para evitar su progresión a obesidad, estado en el que podría iniciarse la agregación de factores de riesgo.

Por su parte, Raina et al. (28), también encontraron un alto grado de asociación entre ambas variables, a pesar de evidenciar una menor prevalencia de obesidad en más de 2000 sujetos adultos de una zona rural de la India. Los mecanismos involucrados en esta relación abarcan la activación del sistema nervioso simpático, del sistema renina-angiotensina-aldosterona, un aumento en la reabsorción de sodio, una progresiva disfunción endotelial e insulinorresistencia; los cuales representan blancos terapéuticos a considerar en la instauración de la terapia farmacológica ${ }^{(29)}$.

Sin embargo, más allá del uso de fármacos antihipertensivos, es importante destacar el papel de los cambios en el estilo de vida no solo como factor involucrado en el control de las cifras de PA sino también en su aparición. Específicamente, los hábitos nutricionales, los cuales en nuestra población parecen relacionarse con HTA de forma independiente al desarrollo de obesidad, lo cual confirma la importancia de los hábitos psicobiológicos en la prevalencia y prevención de esta enfermedad evidenciada en reportes previos ${ }^{(30,31)}$.

Al igual de lo planteado por Mathew et al. ${ }^{(32)}$, en una población hindú, el consumo calórico excesivo constituye en la ciudad de Cuenca, un factor íntimamente relacionado al desarrollo o precipitación de la HTA, por lo cual resulta necesario establecer políticas en salud orientadas a informar a los profesionales de la salud y población general que un consumo calórico diario superior a las 2500 calorías se relaciona a un mayor riesgo de presentar cifras elevadas de PA.

Entre las limitaciones respectivas al tipo de estudio realizado, el diseño transversal imposibilita establecer relaciones de causalidad, para lo cual se necesitan futuros análisis prospectivos que realicen seguimiento de estos sujetos y de los factores de riesgo cardiovascular potenciales que pueden agregarse en caso de no seguir las medidas terapéuticas adecuadas, así como de los potenciales factores que influyen en el adherencia al tratamiento.

En conclusión, los principales factores asociados a la presencia de HTA en la población adulta de la ciudad de Cuenca, Ecuador, fueron la edad, la presencia del antecedente familiar, obesidad y un alto consumo calórico, los cuales deben considerarse y evaluarse de forma sistémica en los pacientes adultos que consulten en nuestra localidad, con el fin de brindar un diagnóstico temprano y un tratamiento integral desde etapas tempranas.

Contribuciones de autoría: RO participó en la concepción y diseño del artículo, recolección de resultados, aprobación de su versión final. MT, WS participaron en recolección de resultados, redacción del artículo, aprobación de la versión final. RA, JS participaron en el análisis e interpretación de datos, asesoría estadística, aprobación de su versión final. JR análisis e interpretación de datos, revisión crítica, aprobación de su versión final. VB participo en la concepción y diseño del artículo, revisión crítica, aprobación de su versión final.

Fuentes de financiamiento: autofinanciado

Conflictos de interés: los autores declaran no tener conflictos de interés.

\section{REFERENCIAS BIBLIOGRÁFICAS}

1. Mozaffarian D, Benjamin EJ, Go AS, Arnett DK, Blaha MJ, Cushman $\mathrm{M}$, et al. Heart Disease and Stroke Statistics-2015 Update: a report from the American Heart Association. Circulation. 2015;131(4):e29-322. doi: 10.1161/CIR.0000000000000152.

2. Ministerio de Salud Pública del Ecuador. Coordinación General de Desarrollo Estratégico en Salud 2012. Datos esenciales de salud: Una mirada a la década 2000-2010 [internet]. Quito: Ministerio de Salud Pública de Ecuador; 2013 [Citado el 19 de noviembre de 2015]. Disponible en http://www.salud.gob.ec/wp-content/
uploads/downloads/2013/05/Datosesenciales-de-salud-2000-2010.pdf.

3. Ortiz-Benavides R, Ortiz-Benavides A, Villalobos M, Rojas J, Torres-Valdez M, Siguencia-Cruz W, et al. Prevalencia de hipertensión arterial en individuos adultos de las parroquias urbanas de la ciudad de Cuenca, Ecuador. Síndrome Cardiometabólico. 2014;4(1):10-21.

4. Beevers G, Lip GY, O’Brien E. ABC of hypertension: The pathophysiology of hypertension. BMJ. 2001;322(7291):912-6.

5. Jolly SE, Koller KR, Metzger JS, Day GM, Silverman A, Hopkins SE, et al. Prevalence of Hypertension and Associated Risk Factors in Western Alaska Native People: The Western Alaska Tribal Collaborative for Health (WATCH) Study. J Clin Hypertens (Greenwich). 2015;17(10):812-8. doi: 10.1111/jch.12483.

6. Ibekwe RU. Modifiable Risk factors of Hypertension and Socio-demographic Profile in Oghara, Delta State; Prevalence and Correlates. Ann Med Health Sci Res. 2015;5(1):71-77. doi: $10.4103 / 2141-9248.149793$

7. Schmieder RE, Ruilope LM. Blood pressure control in patients with 
comorbidities. J Clin Hypertens (Greenwich). 2008;10(8):624-31.

8. Méndez-Castellano $\mathrm{H}$, de Méndez MC. Estratificación social y biología humana: método de Graffar modificado. Arch Ven Pueric Pediatr. 1986;49(3/4):93-104.

9. Ministerio de Sanidad, Servicios Sociales e Igualdad. Encuesta Nacional de Salud. España 2011/12. Serie Informes monográficos $\mathrm{n}^{\circ} 1$. Consumo de alcohol [internet]. Madrid: Ministerio de Sanidad, Servicios Sociales e Igualdad; 2013 [Citado el 19 de noviembre de 2015]. Disponible en: http://www.msssi. gob.es/estadEstudios/estadisticas/ encuestaNacional/encuestaNac2011/ informes Monograficos/ E $\quad N \quad \begin{array}{llllllllll} & \mathrm{N} & \mathrm{E} & 2 & 0 & 1 & 1 & - & 2\end{array}$ $\mathrm{M} O \mathrm{~N} O \mathrm{G}$ R A F I C O $-1_{-}^{-}$ ALCOHOL4.pdf

10. Sicras-Mainar A, Díaz-Cerezo $S$, de Burgoa VS, Navarro-Artieda R. Cost and clinical consequences of smoking cessation in outpatients after cardiovascular disease: a retrospective cohort study. Clinicoecon Outcomes Res. 2013;5:419-27. doi: 10.2147/ CEOR.S43256.

11. International Physical Activity Questionnaire (IPAQ). Guidelines for data processing and analysis of the International Physical Activity Questionnaire (IPAQ)-Short and Long Forms [internet]. 2005 [Citado el 19 de noviembre de 2015]. Disponible en: https://sites.google.com/site/ theipaq/scoring-protocol

12. Sisson SB, Camhi SM, Church TS, Martin CK, Tudor-Locke C, Bouchard $\mathrm{C}$, et al. Leisure time sedentary behavior, occupational/domestic physical activity and metabolic syndrome in U.S. men and women. Metab Syndr Relat Disord. 2009;7(6):529-36. doi: 10.1089/met.2009.0023.

13. World Health Organization. Obesity: preventing and managing the global epidemic. Report of a WHO Consultation (WHO Technical Report Series; 894). Geneva: OMS; 2000.

14. National Center for Health Statistics. Centers for Disease Control and Prevention. The Third National Health and Nutrition Examination Survey (NHANES III, 1988-94) Reference Manuals and Reports [CD-ROM]. Hyattsville: CDC; 1996.

15. Chobanian AV, Bakris GL, Black HR, Cushman WC, Green LA, Izzo JL $\mathrm{Jr}$, et al. Seventh report of the Joint
National Committee on Prevention, Detection, Evaluation, and Treatment of High Blood Pressure. Hypertension. 2003;42(6):1206-52. doi: 10.1161/01. HYP.0000107251.49515.c2

16. Alberti KG, Eckel RH, Grundy SM, Zimmet PZ, Cleeman JI, Donato $\mathrm{KA}$, et al. Harmonizing the Metabolic Syndrome: A Joint Interim Statement of the International Diabetes Federation Task Force on Epidemiology and Prevention: National Heart, Lung, and Blood Institute; American Heart Association; World Heart Federation; International Atherosclerosis Society; International Association for the Study of Obesity. Circulation. 2009;120(16):1640-5. doi: 10.1161/ CIRCULATIONAHA.109.192644.

17. Oparil S, Zaman MA, Calhoun DA. Pathogenesis of Hypertension. Ann Intern Med. 2003;139(9):761-76.

18. Mazzaglia G, Ambrosioni E, Alacqua M, Filippi A, Sessa E, Immordino V, et al. Adherence to antihypertensive medications and cardiovascular morbidity among newly diagnosed hypertensive patients. Circulation. 2009;120(16):1598-605. doi: 10.1161/ CIRCULATIONAHA.108.830299.

19. Jani B, Rajkumar C. Ageing and vascular ageing. Postgrad Med J. 2006; 82(968): 357-62.

20. Pérez-Fernández R, Mariño AF, Cadarso-Suarez C, Botana MA, Tome MA, Solache I, et al. Prevalence, awareness, treatment and control of hypertension in Galicia (Spain) and association with related diseases. J Hum Hypertens. 2007;21(5):366-73. doi:10.1038/sj.jhh.1002158

21. Damasceno A, Azevedo A, SilvaMatos C, Prista A, Diogo D, Lunet N. Hypertension prevalence, awareness, treatment, and control in mozambique: urban/rural gap during epidemiological transition. Hypertension. 2009;54(1):77-83. doi: 10.1161/ HYPERTENSIONAHA.109.132423.

22. Lee HS, Park YM, Kwon HS, Lee JH, Park YJ, Lim SY, et al. Prevalence, awareness, treatment, and control of hypertension among people over 40 years old in a rural area of South Korea: The Chungju Metabolic Disease Cohort (CMC) Study. Clin Exp Hypertens. 2010;32(3):166-78. doi: $10.3109 / 10641960903254497$.

23. Bermúdez VJ, Rojas J, Añez R, Salazar J, Martínez MS, Calvo MJ, et al. Prevalence, awareness, management of hypertension and association with metabolic abnormalities: the Maracaibo city metabolic syndrome prevalence study. Revista Latinoamericana de Hipertensión. 2012;7(4):71-79.

24. Echouffo-Tcheugui JB, Batty GD, Kivimäki M, Kengne AP. Risk models to predict hypertension: a systematic review. PLoS One. 2013;8(7):e67370. doi: 10.1371/journal.pone.0067370.

25. Zhou L, Chen Y, Sun N, Liu X. Family history of hypertension and arterial elasticity characteristics in healthy young people. Hypertens Res. 2008;31(5):8339. doi: 10.1291/hypres.31.833.

26. Goldstein IB, Shapiro D, Weiss RE. How family history and risk factors for hypertension relate to ambulatory blood pressure in healthy adults. J Hypertens. 2008;26(2):276-83. doi: 10.1097/HJH.0b013e3282f15c27.

27. Amira CO, Sokunbi DOB, Sokunbi A. The prevalence of obesity and its relationship with hypertension in an urban community: Data from world kidney day screening programme. International Journal of Medicine and Biomedical Research. 20012;1(2):10410. doi: $10.14194 /$ ijmbr.124

28. Raina DJ, Jamwal DS. Prevalence Study of Overweight/Obesity and Hypertension Among Rural Adults. JK science. 2009;11(1):20-23.

29. Landsberg L, Aronne LJ, Beilin LJ, Burke V, Igel LI, Lloyd-Jones D, et al. Obesity-related hypertension: pathogenesis, cardiovascular risk, and treatment: a position paper of The Obesity Society and the American Society of Hypertension. J Clin Hypertens (Greenwich). 2013;15(1):14-33. doi: 10.1111/jch.12049.

30. Geleijnse JM, Grobbee DE, Kok FJ. Impact of dietary and lifestyle factors on the prevalence of hypertension in Western populations. J Hum Hypertens. 2005;19 Suppl 3:S1-4.

31. Srinath Reddy K, Katan MB.Diet, nutrition and the prevention of hypertension and cardiovascular diseases. Public Health Nutr. 2004;7(1A):167-86.

32. Mathew S, Chary TM. Association of dietary caloric intake with blood pressure, serum lipids and anthropometrics indices in patients with hypertension. Indian J Biochem Biophys. 2013;50:467-73.

Correspondencia: Rina Elizabeth Ortiz Benavides

Dirección: Saraurco y Allcuquiro Totoracocha Cuenca Ecuador

Teléfono: (+593)72864750; (+593) 72864750

Correoelectrónico:rinaortiz@hotmail.es 